\title{
Comportamento ingestivo de vacas primíparas das raças Guzerá e Sindi recebendo dietas com diferentes níveis de ureia
}

\author{
Ingestive behavior of cows primiparous of the Guzera and Sindi breed receiving differents diets
}

\author{
Francisco Helton Sá de Lima $^{\mathrm{I}}$ Severino Gonzaga Neto ${ }^{\mathrm{I}}$ Saulo Vilarim Farias Leite ${ }^{\mathrm{II}}$ \\ Airon Aparecido Silva de Melo ${ }^{\mathrm{III}}$ José Emannuel Lima Sousa ${ }^{\mathrm{IV}}$ José Fábio Paulino de Moura $^{\mathrm{I}}$ \\ José Sidnei Bezerra Lima ${ }^{\mathrm{IV}}$ Thiago Palmeira da Costa ${ }^{\mathrm{IV}}$
}

\section{RESUMO}

\begin{abstract}
Objetivou-se com este trabalho avaliar parâmetros de comportamento ingestivo de vacas primíparas das raças Guzerá e Sindi recebendo dietas com diferentes níveis de inclusão de ureia, em substituição ao farelo de soja, e da cana-de-açúcar, em substituição ao capim elefante. Foram utilizadas oito vacas primíparas, sendo quatro da raça Guzerá e quatro da raça Sindi em lactação, distribuídas em dois quadrados latinos $4 \times 4$. Os tratamentos experimentais consistiram em: T1 (0,0 canade-açúcar $+0,0 \%$ ureia $), T 2$ (13,0\% cana-de-açúcar $+0,6 \%$ ureia), T3 (26,2\% cana-de-açúcar $+1,2 \%$ ureia) e T4 (39,4\% cana-de-açúcar $+1,8 \%$ ureia), na matéria seca. Os animais foram observados a cada cinco minutos, durante 24 horas, para determinação do tempo despendido em alimentação, ruminação e ócio. Não foi observada diferença significativa $(P>0,05)$ para os tempos médios despendidos com as variáveis comportamentais alimentação, ruminação e ócio, à medida que foram aumentados os níveis de inclusão da ureia e cana-de-açúcar nas dietas. $O$ tempo de mastigação total foi maior para os animais da raça Sindi, quando comparado ao dos animais da raça Guzerá. Animais da raça Guzerá apresentaram maior eficiência de alimentação em ( $g$ $M S / h)$ e ruminação, quando expressa em $\left(g M S h^{-1}\right)$ e $\left(g F D N h^{-1}\right)$, quando comparada com a raça Sindi. À medida que se aumentam os níveis de inclusão da ureia e cana de açúcar na dieta, há um decréscimo da eficiência de ruminação expresso em $\left(g F D N h^{-1}\right)$.
\end{abstract}

Palavras chave: cana-de-açúcar, eficiência de alimentação, ócio, ruminação.

\section{ABSTRACT}

This study aims to evaluate the ingestive behavior parameters of primiparous cows Sindi and Guzerá breeds under diet of different levels of urea inclusion replacing the soybean bran and the sugar cane replacing the elephant grass. It has been used eight primiparous cows, being four Guzerá breed and four Sindi lactating, distributed into two Latin squares $4 \times 4$. The experimental treatments consisted of: $T 1$ (0.0 sugar cane $+0.0 \%$ urea $), T 2(13.0 \%$ sugar cane $+0.6 \%$ urea $), T 3(26.2 \%$ sugar cane + $1.2 \%$ urea) and T4 (39.4\% sugar cane $+1.8 \%$ urea), in the dry matter. The animals have been observed each five minutes, during 24 hours, to determine the time spent in feeding, rumination and leisure. There was no significant difference, for the medium time spent with the behavioral variables feeding, rumination and leisure, as it has been increased the levels of urea and sugar cane inclusion in the diets. The total chewing time has been higher for the Sindi cattle when compared with Guzerá. The different levels of sugar cane and urea inclusion have not influenced in the time spent in feeding, rumination and leisure. Animals of the Guzerá breed have shown higher efficiency of feeding in $(g \mathrm{Ms} / \mathrm{h})$ and rumination, when expressed in $\left(g M s h^{-1}\right)$ and $\left(g F D N h^{-1}\right)$, when compared with Sindi breed. As the sugar cane and urea levels increase in the diet, there is a decrease of the rumination efficiency expressed in $\left(g F D N h^{-1}\right)$.

Key words: feeding efficiency, leisure, rumination, sugarcane.

\section{INTRODUÇÃO}

$\mathrm{Na}$ exploração de ruminantes, a alimentação é responsável por grande parte dos custos de produção de uma propriedade leiteira. Tornandose, assim, de fundamental importância conhecer as

'Programa de Doutorado Integrado em Zootecnia, Universidade Federal da Paraíba (UFPB), 58397-000, Areia, PB, Brasil. Email: heltonzootecnia@yahoo.com.br. *Autor para correspondência.

"Departamento de Zootecnia, Universidade Federal Rural do Pernambuco (UFRPE), Unidade Acadêmica de Garanhuns, Garanhuns, PE, Brasil.

II'Empresa Estadual de Pesquisa Agropecuária da Paraíba S. A. (EMEPA), Alagoinha, PB, Brasil.

${ }^{\text {Iv }}$ Programa de Pós Graduação em Zootecnia, UFPB, Areia, PB, Brasil. 
características dos alimentos e seu balanceamento nas dietas, as quais devem ser formuladas para suprir as necessidades dos animais (SILVA et al., 2001). Com isso, a busca por estratégias que diminuam os gastos sem interferir negativamente na produção são frequentes. A utilização de fontes de nitrogênio não proteico, dentre as quais a forma mais comum é a ureia, torna-se uma das alternativas viáveis para o alcance desses objetivos (EZEQUIEL et al., 2001).

Várias fontes de proteína estão disponíveis no mercado, destacando-se o farelo de soja, um dos principais alimentos proteicos disponíveis para a alimentação de bovinos, com excelente composição e sem restrição de uso. Porém um dos principais entraves na utilização do farelo de soja é o alto preço, implicando aumento no custo de produção. Por outro lado, a ureia é uma fonte de nitrogênio não proteico, largamente utilizada na alimentação de ruminantes. A grande vantagem de sua utilização advém do fato de que o ruminante, através dos microrganismos do rúmen, pode satisfazer suas exigências em proteína a partir do nitrogênio não proteico, quando o nível de produção é moderado.

Visando a obter alternativa técnica e economicamente viável, foi proposta a utilização da cana-de-açúcar e ureia, para vacas em lactação das raças Guzerá e Sindi, em relação à recomendação tradicional da região, quando se utiliza o capim elefante com farelo de soja. As alternativas propostas consistem na redução do teor de capim elefante e farelo de soja na dieta, objetivando obter na dieta com cana-de-açúcar e ureia um teor de proteína bruta semelhante ao normalmente encontrado na dieta base.

É notável que alguns genótipos convivem melhor com as condições edafoclimáticas da região semi-árida do que outros. Pesquisas realizadas no nordeste brasileiro com a raça Sindi, por exemplo, reportam altos índices reprodutivos (MARIZ, 1987), melhor tolerância ao calor (SOUZA, 2003) e menores exigências nutricionais (SILVA, 2006). Já MACARI \& OLIVEIRA (2003) relatam que a raça Guzerá possui material genético apropriado para as regiões brasileiras onde as adversidades ecológicas são maiores.

O impacto econômico positivo do zebuíno para a pecuária brasileira é consideravelmente superior ao do material genético de raças europeias, à medida que ele proporciona a instalação no país de uma pecuária auto sustentável, não dependente de fatores externos e nem da modificação constante do meioambiente natural que se dispõe (JOSAHKIAN, 2000).

No Nordeste brasileiro, poucos estudos foram desenvolvidos com as raças Guzerá e Sindi no intuito de avaliar o comportamento ingestivo e práticas de manejo alimentar, visando a uma maior eficiência na produção desses rebanhos. $\mathrm{O}$ entendimento do comportamento ingestivo dos animais, como ferramenta útil à adequação do manejo dos rebanhos, se faz necessário, sendo este intrinsecamente relacionado à produção. Assim, objetivou-se, com o presente estudo, avaliar parâmetros de comportamento ingestivo de vacas primíparas das raças Guzerá e Sindi recebendo dietas com diferentes níveis de ureia, em substituição ao farelo de soja, e de cana-de-açúcar, em substituição ao capim elefante.

\section{MATERIAL E MÉTODOS}

O experimento foi conduzido na Estação Experimental de Alagoinha, pertencente à Empresa Estadual de Pesquisa Agropecuária da Paraíba S.A. - Emepa, localizada na Mesorregião Agreste Paraibano, Microrregião de Guarabira, no Município de Alagoinha, PB, nas coordenadas geográficas $6^{\circ} 57^{\prime}$ 00 " $\mathrm{S}$ e $35^{\circ} 32$ ' 42 " W. Gr., com altitude de $135 \mathrm{~m}$. As precipitações pluviais atingem uma média de 995mm anuais. As temperaturas máximas e mínimas registradas durante o período experimental foram 32 e $26^{\circ} \mathrm{C}$, respectivamente. A umidade relativa máxima foi $77 \%$ e a mínima $39 \%$.

O ensaio experimental foi desenvolvido no período de janeiro a março de 2006 tendo uma duração de 60 dias, divididos em quatro períodos experimentais de 15 dias.

Foram utilizadas oito vacas primíparas em lactação, sendo quatro da raça Guzerá e quatro da raça Sindi, com peso vivo médio de $455 \mathrm{~kg} \pm 30 \mathrm{~kg}$ e $265 \mathrm{~kg} \pm 11 \mathrm{~kg}$, respectivamente, distribuídas aleatoriamente em dois quadrados latinos $4 \times 4$, e alojadas em baias individuais $2,5 \times 2,5 \mathrm{~m}$, contendo comedouro e bebedouro para o controle do consumo de alimento e ingestão de água.

As dietas foram formuladas para serem isoproteicas e apresentavam capim elefante (Penissetum purpureum), cana-de-açúcar (Sacarum officinarum), farelo de milho, farelo de soja, caroço de algodão, ureia e suplemento mineral, contendo diferentes níveis de inclusão da ureia em substituição ao farelo de soja e de cana-de-açúcar em substituição ao capim elefante, distribuídas nos seguintes tratamentos: T1 (0,0 cana-de-açúcar $+0,0 \%$ ureia), T2 (13,0\% cana-de-açúcar $+0,6 \%$ ureia), T3 (26,2\% cana-de-açúcar $+1,2 \%$ ureia) e T4 $(39,4 \%$ cana-deaçúcar $+1,8 \%$ ureia), na matéria seca.

As dietas foram fornecidas duas vezes ao dia, às 8 h e às $16 \mathrm{~h}$, na forma de ração completa, sendo 
que $40 \%$ do consumo diário foi ofertado pela manhã e $60 \%$ à tarde, foram permitidas sobras de $10 \%$ do total do alimento fornecido no dia, para garantir o consumo à vontade. $\mathrm{O}$ alimento volumoso (capim elefante e cana-de-açúcar) e o concentrado foram pesados separadamente e misturados no momento do fornecimento. As proporções e composições das dietas experimentais estão na tabela 1.

As variáveis comportamentais observadas e registradas foram: alimentação, ruminação e ócio, determinadas no $15^{\circ}$ dia de cada período experimental através da quantificação dos intervalos de tempo. No registro do tempo despendido com as variáveis, adotou-se a observação visual dos animais a cada cinco minutos por 24 horas. As atividades desenvolvidas pelos animais foram registradas em etograma individual, totalizando 288 registros por animal e por período. Os eventos que constituem o comportamento ingestivo foram considerados como mutuamente excludentes, ou seja, a cada registro, cada um dos animais foi classificado em apenas uma atividade: alimentação, ruminação e ócio. O tempo total despendido em cada atividade foi calculado multiplicando-se o número total de observações por cinco de acordo com a metodologia de BÜRGER et al. (2000).
Durante a observação noturna, as baias permaneceram sem iluminação ambiente, sendo necessário a utilização de lanternas para observar a ocorrência dos eventos.

A eficiência de alimentação (EAL), a eficiência de ruminação (ERU) e o tempo de mastigação total (TMT, $\mathrm{h}$ dia $^{-1}$ ) foi determinado como sendo o somatório do tempo de alimentação e ruminação (TAL + TRU), segundo a metodologia descrita por BÜRGUER et al. (2000).

Os resultados referentes aos fatores do comportamento ingestivo foram obtidos utilizandose as equações: $\mathrm{EAL}=\mathrm{CMS} / \mathrm{TAL}$; $\mathrm{ERU}=\mathrm{CMS} / \mathrm{TRU}$; ERU $=$ CFDN/TRU e TMT $=$ TAL+TRU, em que: $E A L$ $\left(\mathrm{g} \mathrm{MS} \mathrm{h}^{-1}\right)$ é eficiência de alimentação; CMS (g MS dia $^{-1}$ ), consumo de MS; CFDN (g FDN dia ${ }^{-1}$ ), consumo de FDN; TAL (h dia ${ }^{-1}$ ), tempo de alimentação; ERU $\left(\mathrm{g} \mathrm{MS} \mathrm{h}^{-1} ; \mathrm{g} \mathrm{FDN} / \mathrm{h}\right.$ ), eficiência de ruminação; TRU $\left(\mathrm{h} \operatorname{dia}^{-1}\right)$, tempo de ruminação; TMT (h dia $\left.{ }^{-1}\right)$, tempo de mastigação total.

Os dados foram avaliados mediante análises de variância e regressão. Para comparação das médias entre raças, foi usado o teste $\mathrm{F}$, ao nível de $5 \%$ de probabilidade, empregando-se o Sistema de Análises Estatísticas e Genéticas - SAEG (UFV, 1999).

Tabela 1 - Proporções de ingredientes e composição bromatológica das dietas experimentais, com base na matéria seca.

\begin{tabular}{|c|c|c|c|c|}
\hline \multirow{2}{*}{ Ingredientes $(\%)$} & \multicolumn{4}{|c|}{ veis de Inclusão de Ureia-- } \\
\hline & 0,0 & 0,6 & 1,2 & 1,8 \\
\hline Capim & 70,00 & 59,50 & 49,00 & 38,50 \\
\hline Cana de açúcar & 0,00 & 13,20 & 26,20 & 39,60 \\
\hline Farelo de milho & 3,60 & 3,60 & 3,60 & 3,70 \\
\hline Farelo de soja & 16,80 & 13,40 & 10,30 & 6,70 \\
\hline Caroço de algodão & 8,40 & 8,40 & 8,40 & 8,40 \\
\hline Ureia & 0,00 & 0,60 & 1,20 & 1,80 \\
\hline Suplemento mineral & 1,20 & 1,30 & 1,30 & 1,30 \\
\hline \multicolumn{5}{|l|}{ Nutriente } \\
\hline $\operatorname{MS}^{1}(\%)$ & 35,80 & 34,49 & 32,81 & 30,61 \\
\hline $\mathrm{MM}^{1}$ & 8.27 & 8,36 & 6,71 & 8,22 \\
\hline $\mathrm{MO}^{1}$ & 91,72 & 91,63 & 93,28 & 91,77 \\
\hline $\mathrm{PB}^{1}$ & 12,30 & 12,70 & 12,47 & 12,55 \\
\hline $\mathrm{EE}^{1}$ & 3,67 & 3,57 & 3,48 & 3,39 \\
\hline $\mathrm{FDN}^{1}$ & 60,63 & 59,02 & 57,51 & 55,97 \\
\hline $\mathrm{FD} \wedge^{1}$ & 36,35 & 36,37 & 36,52 & 36,65 \\
\hline $\mathrm{CNF}^{1}$ & 16,30 & 17,46 & 18,43 & 19,62 \\
\hline $\mathrm{CHO}^{1}$ & 75,75 & 75,37 & 77,33 & 75,83 \\
\hline $\mathrm{NDT}^{1}$ & 58,08 & 58,53 & 58,88 & 59,15 \\
\hline
\end{tabular}

${ }^{1} \%$ na Matéria Seca (MS).

$\mathrm{MM}=$ Matéria Mineral, $\mathrm{MO}=$ Matéria Orgànica, $\mathrm{PB}=$ Proteína Bruta, EE = Extrato Etéreo, FDN = Fibra em Detergente Neutro, FDA = Fibra em Detergente Ácido, CNF - Carboidratos Não Fibrosos, CHO - Carboidratos Totais, NDT - Nutrientes Digestíveis Totais.

Ciência Rural, v.43, n.4, abr 2013. 


\section{RESULTADOS E DISCUSSÃO}

Não foi observada diferença significativa $(\mathrm{P}>0,05)$ com o aumento nos níveis de ureia $\mathrm{e}$ cana-de-açúcar em substituição ao farelo de soja e capim elefante, para os tempos médios despendidos com alimentação, ruminação e ócio, em ambos os genótipos estudados, tabela 2. Observou-se um maior tempo total despendido com alimentação para vacas da raça Sindi de 30,99\% (7 horas e 44 minutos), ao passo que, para vacas da raça Guzerá, este evento representou $27,02 \%$ (6 horas e 48 minutos), em relação ao tempo de total de observação diária. Uma possível explicação para esse comportamento pode ser atribuída às características anatômicas das vacas Sindi, uma vez que esses animais consomem menor quantidade de alimento por bocado, necessitando, assim, de maior tempo de alimentação para atender as suas necessidades nutricionais.

Não houve efeito de raça nem dos níveis de inclusão de ureia e cana-de-açúcar em substituição ao farelo de soja e capim elefante sobre o tempo despendido em ruminação, devendo-se, provavelmente, ao fato de as dietas experimentais apresentarem teores de fibra em detergente neutro semelhantes.

PEREIRA et al. (2007) observaram diferença quanto ao tempo médio dispendido em ruminação, trabalhando com novilhas alimentadas com dietas à base de capim-elefante com dois níveis de fibra em detergente neutro (FDN) 30 e $60 \%$. Sendo os tempos de ruminação maiores quando se aumenta o teor de FDN da dieta. Em contrapartida, ocorreu uma diminuição no tempo despendido com o ócio, demonstrando a importância desse ingrediente nas dietas em relação ao comportamento dos animais. Esses resultados diferem dos encontrados neste estudo devido à semelhança no teor de FDN das dietas avaliadas.

O ócio é a atividade que não inclui nem ruminação nem alimentação, sendo observado maior tempo total despendido para esta atividade nas vacas Guzerá $32,73 \%$ (8 horas e 25 minutos), quando comparada com a raça Sindi, que apresentou média de tempo despendido em ócio de 26,89\% (6 horas e 45 minutos). Esse comportamento para vacas da raça Sindi pode ter ocorrido em virtude de os animais terem despendido um maior tempo se alimentando. Maiores médias de tempos despendidos com a atividade ócio (10h58min à $12 \mathrm{~h} 13 \mathrm{~min})$ foram encontradas por MENDONÇA et al. (2004), trabalhando com vacas holandesas alimentadas com dietas à base de canade-açúcar e ureia, provavelmente, esses resultados estão associados à relação volumoso:concentrado, empregada neste estudo.

Observam-se maiores médias de ingestão de matéria seca para vacas Guzerá, quando comparada com as vacas Sindi em todos tratamentos experimentais avaliados. Esse fato pode ser explicado devido ao maior tamanho corporal apresentado por esta raça. $\mathrm{O}$ consumo de matéria seca reduziu linearmente $13,8 \%$ com a inclusão de ureia e cana-deaçúcar na dieta para animais da raça Guzerá, ocorrendo um decréscimo de $0,9 \mathrm{~kg}$ de matéria seca ingerida a cada unidade de inclusão desses componentes. Para vacas Sindi, observou-se comportamento semelhante com um efeito linear decrescente $(\mathrm{P}<0,05)$ de acordo com o aumento dos níveis de inclusão de ureia nas dietas, apresentando uma variação de $22,5 \%$ entre os tratamentos experimentais.

O menor consumo de matéria seca foi provavelmente causado pelos efeitos metabólicos da ureia e, ou, devido a pouca palatabilidade ao alimento, à medida que se elevou o teor de ureia na ração,

Tabela 2 - Médias, coeficientes de variação (CV), determinação $\left(r^{2}\right)$ e equação de regressão para os tempos despendidos em minutos, com alimentação, ruminação e ócio, em animais das raças Guzerá (G) e Sindi (S), em função das dietas experimentais.

\begin{tabular}{|c|c|c|c|c|c|c|c|c|c|}
\hline \multirow{2}{*}{ Variáveis } & \multirow{2}{*}{ Raça } & \multicolumn{4}{|c|}{---------Níveis de Inclusão de Ureia---------- } & \multirow{2}{*}{ Média } & \multirow{2}{*}{ Equação de regressão } & \multirow{2}{*}{$\mathrm{CV} \%$} & \multirow{2}{*}{$r^{2}$} \\
\hline & & 0,0 & 0,6 & 1,2 & 1,8 & & & & \\
\hline \multirow{2}{*}{ Alimentação } & G & 371,25 & 436,25 & 378,75 & 370,00 & $389,06 \mathrm{~B}$ & $\hat{y}=389,06$ & 9,66 & -- \\
\hline & $\mathrm{S}$ & 450,00 & 468,75 & 426,25 & 440,00 & $446,25 \mathrm{~A}$ & $\hat{y}=446,25$ & 9,66 & -- \\
\hline \multirow{2}{*}{ Ruminação } & G & 580,00 & 611,25 & 557,50 & 570,00 & 579,69 & $\hat{y}=579,69$ & 10,05 & -- \\
\hline & $\mathrm{S}$ & 613,75 & 607,5 & 606,25 & 598,75 & 606,56 & $\hat{y}=606,56$ & 10,05 & -- \\
\hline \multirow{2}{*}{ Ócio } & G & 488,75 & 392,50 & 503,75 & 500,00 & $471,25 \mathrm{~A}$ & $\widehat{y}=471,25$ & 18,19 & -- \\
\hline & $\mathrm{S}$ & 376,25 & 363,75 & 407,5 & 401,25 & $387,19 \mathrm{~B}$ & $\widehat{y}=387,19$ & 18,19 & -- \\
\hline
\end{tabular}

Médias com letras iguais na coluna para cada variável não diferem entre si pelo Teste $\mathrm{F}$ a $5 \%$ de probabilidade.

Ciência Rural, v.43, n.4, abr, 2013. 
conforme HUBER \& COOK (1972), que atribuíram a redução no consumo em dietas com alto nível de ureia no concentrado (1 a 3\%) a pouca palatabilidade desta, e não a efeitos ruminais ou pós-ruminais.

O consumo de fibra em detergente neutro expresso em $\mathrm{kg} / \mathrm{dia}$ diminuiu linearmente $(\mathrm{P}<0,05)$, para vacas Guzerá e Sindi, com a inclusão de ureia e cana-deaçúcar nas dietas, refletindo comportamento semelhante ao observado para a matéria seca. Esse comportamento pode ter ocorrido em consequência da redução no consumo de matéria seca, à medida que aumentaram os níveis de inclusão dos ingredientes estudados.

Os resultados encontrados neste estudo corroboram CARVALHO et al. (2011), em que, dietas com teores de FDN similares, mas compostas por alimentos diferentes, podem predispor os animais a diferentes dispêndios nas atividades diárias.

Observou-se diferença significativa entre as raças para a eficiência de alimentação EAL ( $\mathrm{g}$ de $\mathrm{MS}^{-1}$ ), apresentando médias de 1660,96 e 1003,83g de MS h${ }^{-1}$ para vacas Guzerá e Sindi, respectivamente, tabela 3. Vacas Guzerá apresentaram uma melhor eficiência de alimentação, devido a um maior consumo de $g \mathrm{MS} \mathrm{h}^{-1}$, haja vista que as vacas Sindi despenderam um maior tempo com alimentação e apresentaram menor consumo de matéria seca em $\mathrm{kg} \mathrm{dia}^{-1}$.

À medida que se aumentaram os níveis de inclusão da ureia e cana-de-açúcar nas dietas experimentais para as raças Guzerá e Sindi, observouse decréscimo $(\mathrm{P}<0,05)$ na eficiência de ruminação. Esse comportamento pode estar relacionado à redução na taxa de passagem da fibra nos compartimentos ruminais e, consequentemente, ao maior tempo de retenção ruminal da FDN. Diante do exposto, fica claro que, além dos teores de FDN na dieta, a composição dessa fibra também pode influenciar significativamente nas atividades comportamentais, principalmente ruminação.

De acordo com PEREIRA et al. (2004), a eficiência de ruminação é importante no controle da utilização de volumosos e, quando ocorre uma redução nesta eficiência de ruminação, esta não pode ser compensada pelo prolongamento da atividade de ruminação.

O tempo de mastigação total foi maior $(\mathrm{P}<0,05)$ para vacas Sindi, quando comparado com o comportamento observado para vacas Guzerá. Esse fato pode ser justificado devido às vacas

Tabela 3 - Médias, coeficientes de variação (CV), determinação (r2) e equação de regressão para as variáveis, consumo de matéria seca (CMS), consumo de fibra em detergente neutro (CFDN), eficiència de alimentação de MS (EAL), eficiência de ruminação da MS (ERU) e do FDN (ERUFDN), tempo de mastigação total (TMT) para animais das raças Guzerá (G) e Sindi (S), em função das dietas experimentais.

\begin{tabular}{|c|c|c|c|c|c|c|c|c|c|}
\hline \multirow{2}{*}{ Variáveis } & \multirow{2}{*}{ Raça } & \multicolumn{4}{|c|}{--Níveis de Inclusão de Ureia---..-- } & \multirow{2}{*}{ Média } & \multirow{2}{*}{$\begin{array}{l}\text { Equação de } \\
\text { regressão }\end{array}$} & \multirow{2}{*}{$\mathrm{CV} \%$} & \multirow{2}{*}{$r^{2}$} \\
\hline & & 0,0 & 0,6 & 1,2 & 1,8 & & & & \\
\hline \multirow{2}{*}{ CMS $\left(\mathrm{kg} \mathrm{dia}^{-1}\right)$} & G & 11,17 & 10,97 & 10,17 & 9,63 & $10,49 \mathrm{~A}$ & $\hat{y}=11,29-0,900 x$ & 8,89 & 0,95 \\
\hline & $S$ & 8,18 & 7,90 & 7,22 & 6,34 & $7,41 \mathrm{~B}$ & $\hat{y}=8,338-1,033 x$ & 8,89 & 0,95 \\
\hline \multirow{2}{*}{ CFDN $\left(\mathrm{kg} \mathrm{dia}^{-1}\right)$} & G & 7,71 & 7,44 & 6,48 & 6,28 & $6,98 \mathrm{~A}$ & $\hat{y}=7,771-0,876 x$ & 9,25 & 0,95 \\
\hline & $S$ & 5,65 & 5,36 & 4,60 & 4,13 & $4,94 \mathrm{~B}$ & $\hat{y}=5,732-0,884 x$ & 9,25 & 0,97 \\
\hline \multirow{2}{*}{$\mathrm{EAL}\left(\mathrm{g} \mathrm{MS} \mathrm{h}^{-1}\right)$} & $\mathrm{G}$ & 1898,42 & 1535,18 & 1602,40 & 1607,86 & $1660,97 \mathrm{~A}$ & $\hat{y}=1660,96$ & 13,82 & -- \\
\hline & $S$ & 1121,18 & 1014,17 & 1017,00 & 862,97 & $1003,83 \mathrm{~B}$ & $\hat{y}=1003,83$ & 13,82 & -- \\
\hline \multirow{2}{*}{$\operatorname{ERU}\left(\mathrm{g} \mathrm{MS} \mathrm{h}^{-1}\right)$} & $\mathrm{G}$ & 1156,67 & 1074,60 & 1104,66 & 1014,48 & $1087,60 \mathrm{~A}$ & $\hat{y}=1087,60$ & 9,79 & -- \\
\hline & $\mathrm{S}$ & 801,98 & 806,54 & 714,20 & 646,79 & $742,38 \mathrm{~B}$ & $\hat{y}=881,8-5,79 x$ & 9,79 & 0,88 \\
\hline \multirow{2}{*}{ ERU $\left(\mathrm{g} \mathrm{FDN}^{-1}\right)$} & $\mathrm{G}$ & 799,24 & 729,10 & 703,81 & 661,83 & $723,50 \mathrm{~A}$ & $\hat{y}=832,8-3,75 x$ & 9,48 & 0,96 \\
\hline & $S$ & 554,16 & 547,22 & 455,04 & 421,96 & $494,60 \mathrm{~B}$ & $\hat{y}=616,7-8,87 x$ & 9,48 & 0,91 \\
\hline \multirow{2}{*}{ TMT $\quad\left(\mathrm{h} \mathrm{dia}^{-1}\right)$} & $\mathrm{G}$ & 15,85 & 17,46 & 15,60 & 15,67 & $16,15 \mathrm{~B}$ & $\hat{y}=16,15$ & 7,72 & -- \\
\hline & $\mathrm{S}$ & 17,73 & 17,94 & 17,21 & 17,31 & $17,55 \mathrm{~A}$ & $\hat{y}=17,55$ & 7,72 & -- \\
\hline
\end{tabular}

Médias com letras iguais na coluna para cada variável não diferem entre si pelo Teste $\mathrm{F}$ a $\mathbf{5 \%}$ de probabilidade.

Ciência Rural, v.43, n.4, abr 2013. 
Sindi apresentarem maior tempo despendido com alimentação e ruminação. Não houve efeito dos níveis de inclusão de ureia e cana-de-açúcar em substituição ao farelo de soja e capim elefante sobre o tempo de mastigação total.

Segundo os autores DE BOEVER et al. (1990), FISCHER, (1996), animais de menor peso vivo podem apresentar menores taxas de mastigação tanto na ingestão, como para a ruminação, em relação àqueles de maior peso, o que pode provocar o aumento do tempo total diário dedicado a essas atividades, confirmando assim o ocorrido neste estudo.

\section{CONCLUSÃO}

Os níveis de inclusão de ureia e cana-deaçúcar em substituição ao farelo de soja e capim elefante não alteraram as variáveis comportamentais de vacas primíparas das raças Guzerá e Sindi. Animais da raça Guzerá apresentaram maior eficiência de alimentação em (g MS $\mathrm{h}^{-1}$ ) e ruminação, quando expressa em $\left(\mathrm{g} \mathrm{MS} \mathrm{h}^{-1}\right)$ e $\left(\mathrm{g} \mathrm{FDN} \mathrm{h}^{-1}\right)$. À medida que se aumentam os níveis de inclusão da ureia e cana de açúcar na dieta, há um decréscimo da eficiência de ruminação expresso em $\left(\mathrm{g} \mathrm{FDN} \mathrm{h}^{-1}\right)$.

\section{REFERÊNCIAS}

BÜRGER, P.J. al. Comportamento ingestivo em bezerros holandeses alimentados com dietas contendo diferentes níveis de concentrado. Revista Brasileira de Zootecnia, v.29, p.236242, 2000. Disponível em: <http://www.revistasbz.org.br/scripts/ revista/SBZ1/Artigos/2580.PDF>. Acesso em: 10 nov. 2006.

CARVALHO, G.G.P. et al. Comportamento ingestivo em caprinos alimentados com dietas contendo cana-de-açúcar tratada com óxido de cálcio. Revista Brasileira de Zootecnia, v.40, n.8, p.1767-1773, 2011. Disponível em: <http://www.revistasbz.org. br/scripts/revista/SBZ1/Artigos/9175.PDF>. Acesso em: 15 out. 2011. ISSN 1806-9290.

DEBOEVER, J.L. et al. Chewing activity of ruminants as a measure of physical structure - A review of factors affecting it. Animal Feeding Science Technology, v.27, p.281-291, 1990. Disponível em: <http:// ac.els-cdn.com/037784019090143V/1-s2.0-037784019090143Vmain.pdf?_tid=fd9cedfcaa948 adad3df598f49b8424e\&acdn at $=1338312784 \_b b 8$ e38a15c87eaed9cdd0f8f9f6566d2>. Acesso em: 10 nov. 2006.

EZEQUIEL, J.M.B. et al. Digestibilidade aparente da energia e da fibra de dietas para ovinos contendo ureia, amiréia ou farelo de algodão. Revista Brasileira de Zootecnia, v.30; n.1, p.231235, 2001. Disponível em: <http://www.revistasbz.org.br/scripts/ revista/SBZ1/Artigos/2802.PDF>. Acesso em: 15 nov. 2011. ISSN 1806-9290.

FISCHER, V. Efeitos do fotoperíodo, da pressão de pastejo e da dieta sobre o comportamento ingestivo de ruminantes. 1996. 243f. Tese (Doutorado em Zootecnia) - Curso de Pós-graduação em Zootecnia, Universidade Federal do Rio Grande do Sul, RS.

HUBER, J.T.; COOK, R.M. Influence of site of administration of urea on voluntary intake of concentrate by lactating cows. Jounal of Dairy Science, v.55, n.10, p.1470-1473, 1972. Disponível em: <http://download.journals.elsevierhealth.com/ pdfs/journals/0022-0302/PIIS0022030272856960.pdf>. Acesso em: 05 dez. 2006.

JOSAHKIAN, L.A. Programa de melhoramento genético das raças zebuínas. In: SIMPÓSIO NACIONAL DA SOCIEDADE BRASILEIRA DE MELHORAMENTO ANIMAL, 3., 2000, Belo Horizonte, MG. Anais... Belo Horizonte: Sociedade Brasileira de Melhoramento Animal, 2000. p.73-93.

MACARI, T.B.; OLIVEIRA, J.A. de. Análise genético-quantitativa de pesos aos 8, 12, 18 e 24 meses de idade em um rebanho da raça Guzerá. Revista Brasileira de Zootecnia, v.32, n.6, supl.1, p.16041613, 2003. Disponível em: <http://www.revistasbz.org.br/scripts/ revista/SBZ1/Artigos/3846.PDF>. Acesso em: 15 nov. 2011.

MARIZ, F.P. et al. Avaliação da eficiência reprodutiva de bovinos da raça Sindi no sertão da Paraíba. In: REUNIÃO ANUAL DA SOCIEDADE BRASILEIRA DE ZOOTECNIA, 21., 1987, João Pessoa, PB. Anais... João Pessoa: Sociedade Brasileira de Zootecnia, 1987. p.140.

MENDONÇA, S.S. et al. Comportamento ingestivo de vacas leiteiras alimentadas com dietas à base de cana-de-açúcar ou silagem de milho. Revista Brasileira de Zootecnia, v.33, n.3, p.723-728, 2004. Disponível em: <http://www.revistasbz.org.br/scripts/revista/ SBZ1/Artigos/3872.PDF>. Acesso em: 10 jul. 2011.

NATIONAL RESEARCH COUNCIL (NRC). Nutrients requirements of dairy cattle. 7.ed. Washington, DC: National Academy, 2001. 408p.

PEREIRA, E.S. et al. Comportamento ingestivo de vacas em lactação alimentadas com diferentes fontes de volumosos conservados. Semina: Ciências Agrárias. v.25, n.2, p.159166, 2004. Disponível em: <http://www.uel.br/portal/frm/ frmOpcao.php?opcao=http://www.uel.br/revistas/uel/index.php/ semagrarias>. Acesso em: 10 jan. 2007.

PEREIRA, J.C. et al. Comportamento Ingestivo e taxa de passagem de partículas em novilhas leiteiras de diferentes grupos genéticos submetidas a dietas com diferentes níveis de fibra. Revista Brasileira de Zootecnia, v.36, p.2134-2142, 2007. Disponível em: <http://www.revistasbz.org.br/scripts/revista/ SBZ1/Artigos/6158.PDF>. Acesso em: 20 nov. 2007. ISSN online: $1806-9290$.

SILVA, G.L.S. Metabolismo sanguíneo de novilhas sindi submetidas a diferentes níveis de suplementação alimentar na região semi-árida. In: REUNIÃO ANUAL DA SOCIEDADE BRASILEIRA DE ZOOTECNIA, 43., 2006, João Pessoa, PB. Anais... João Pessoa: Sociedade Brasileira de Zootecnia, [2006]. (CD-ROM).

SILVA, R.M.N. et al. Ureia para vacas em lactação. 1. Consumo, digestibilidade, produção e composição do leite1. Revista Brasileira de Zootecnia, v.30, n.5, p.1639-1649, 2001. 
Disponível em: <http://www.revistasbz.org.br/scripts/revista/ SBZ1/Artigos/3098.PDF>. Acesso em: 05 dez. 2006.

SOUZA, B.B. et al. Avaliação da adaptabilidade do gado sindi as condições do semi-árido nordestino. In: REUNIÃO ANUAL DA SOCIEDADE BRASILEIRA DE ZOOTECNIA, 40., 2003,
Campo Grande. Anais... Campo Grande: Sociedade Brasileira de Zootecnia, [2003]. (CD-ROM).

UNIVERSIDADE FEDERAL DE VIÇOSA (UFV). SAEG Sistema para análise estatística e genética, versão 8.0. Viçosa, MG: Fundação Arthur Bernardes, 1999. 